$10-2015$

\title{
Multi-dimensional Glycan Microarrays with Glyco-macroligands
}

\author{
Satya Nandana Narla \\ Cleveland State University \\ Huan Nie \\ Harbin Institute of Technology \\ Yu Li \\ Harbin Institute of Technology \\ Xue-Long Sun \\ Cleveland State University, x.sun55@csuohio.edu
}

Follow this and additional works at: https://engagedscholarship.csuohio.edu/scichem_facpub

Part of the Biochemistry Commons, and the Chemistry Commons

How does access to this work benefit you? Let us know!

Publisher's Statement

The final publication is available at Springer via http://dx.doi.org/10.1007/s10719-015-9580-z

\section{Recommended Citation}

Narla, S. N.; Nie, H.; Li, Y.; Sun, X. Multi-dimensional glycan microarrays with glyco-macroligands.

Glycoconj. J. 2015, 32, 483-495.

This Article is brought to you for free and open access by the Chemistry Department at EngagedScholarship@CSU.

It has been accepted for inclusion in Chemistry Faculty Publications by an authorized administrator of

EngagedScholarship@CSU. For more information, please contact library.es@csuohio.edu. 


\title{
Multi-dimensional glycan microarrays with glyco-macroligands
}

\author{
Satya Nandana Narla ${ }^{1} \cdot \mathrm{Huan} \mathrm{Nie}^{2} \cdot \mathrm{Yu} \mathrm{Li}^{2} \cdot$ Xue-Long Sun ${ }^{1}$
}

Received: 28 January 2015/Revised: 6 March 2015/Accepted: 10 March 2015/Published online: 10 May 2015

(C) Springer Science+Business Media New York 2015

\begin{abstract}
Glycan microarray has become a powerful highthroughput tool for examining binding interactions of carbohydrates with the carbohydrate binding biomolecules like proteins, enzymes, antibodies etc. It has shown great potential for biomedical research and applications, such as antibody detection and profiling, vaccine development, biomarker discovery, and drug screening. Most glycan microarrays were made with monovalent glycans immobilized directly onto the array surface via either covalent or non-covalent bond, which afford a multivalent glycans in two dimensional (2D) displaying. A variety of glyco-macroligands have been developed to mimic multivalent carbohydrate-protein interactions for studying carbohydrate-protein interactions and biomedical research and applications. Recently, a number of glyco-macroligands have been explored for glycan microarray fabrication, in particular to mimick the three dimensional (3D) multivalent display of cell surface carbohydrates. This review highlights these recent developments of glyco-macroligand-based microarrays, predominantly, novel glycan microarrays with glycomacroligands like glycodendrimers, glycopolymers, glycoliposomes, neoglycoproteins, and glyconanoparticles with the effort in controlling the density and orientation of glycans on the array surface, which facilitate both their binding specificity and affinity and thus the high performance of glycan microarrays.
\end{abstract}

Keywords Glycan microarray - Glycodendrimers · Glycopolymers · Glycoliposomes · Neoglycoproteins · Glyconanoparticles

\section{Introduction}

Cell surface carbohydrates, prevailing as glycoconjugates such as glycoproteins, glycolipids and proteoglycans, are involved in many biological processes, such as cell-cell interaction [1, 2] immune recognition events [3], pathogen-host interaction [4], tumor metastasis [5], tissue growth and repair [6] etc. Understanding the interactions between carbohydrates and carbohydrate binding proteins (CBPs) are highly needed for clarifying the molecular mechanisms of these physiological and pathological pathways and discovering new therapeutic and diagnostic principles. Glycan microarrays have been developed and proved as powerful high throughput tools to study the interactions between carbohydrates and carbohydrate binding molecules like proteins [7, 8], antibodies [9], and pathogens [10]. Recently, they have been applied to clinical antibody detection and profiling [11], biomarker discovery [12], and drug screening [13] applications. Nevertheless, many challenges still exist for their potential biomedical research and applications. Mainly, there are two critical limitations that prevent the wide and practical applications of glycan microarray technology. First, the detection is limited by limited epitope availability for microarray fabrication from both isolation from natural sources and synthesis. Both isolation and synthesis of carbohydrates are still very challenging due to their structure complexity, low throughput, intense labor as well as the need of special skills. Second, the features of glycan presentation on the microarray surface such as density and orientation of glycans have substantial effect on protein recognition related to both affinity and specificity. 
Conventional glycan microarrays were developed as two dimensional (2D) glycan ligands with monovalent glycans immobilized directly onto the array surface via either covalent or non-covalent bond [7-10], which affords the multivalent glycans but lacks of the three dimensional (3D) cell surface carbohydrates formats. In addition, there is little control over glycan presentation. Therefore, these glycan arrays usually result in low signal intensity and substantial non-specific binding of target proteins due to an insufficient number of accessible glycans and the presence of surface-protein interactions. To overcome these limitations, recently, glycan density and orientation on the microarray surface has been recognized as an important feature of carbohydrate recognition and thus has been investigated extensively. A recent comprehensive review paper summarized well the most important development of glycan array technologies [14]. Overall, different glycan array platforms produced different results, which is well demonstrated by a comparative analysis of glycan array platforms with six different modes of glycan presentation using five well-known lectins reported by Mahal et al. [15]. As they indicated, multiple microarray platforms may be required to obtain the best understanding of glycan binding in a system and much microarray development remains to get a comprehensive view of glycan-binding protein specificity.

In nature, carbohydrate receptors exist mostly as glycoconjugates such as glycoproteins and glycolipids, which present multivalent carbohydrates either in a single glycoconjugate or by combining multiple glycoconjugates as a cluster. In addition, CBPs contain two or more carbohydrate binding sites or assemble into oligomers with multiple binding sites, which allow them to simultaneously bind two or more carbohydrates and facilitate functional affinity (avidity) and selectivity. Therefore, multivalent carbohydrate ligand presentation has been a central theme to study and apply intricate carbohydrate-protein interactions. In the past decades, various glycopolymeric molecules have been investigated for studying carbohydrate-protein interactions and biomedical research and applications, such as for multivalent inhibitors and antagonists development. For example, different multivalent carbohydrate molecules like glycodendrimers [16-22], glycopolymers [23-28], glycoliposomes [29-32], neoglycoproteins [11, 13,33], and glyconanoparticles [34] as glyco-macroligands were developed to attain more specific and strong carbohydrate-protein interactions. It is acknowledged that not only the multivalency of the carbohydrates affect the binding specificity but also the orientation of the carbohydrates on the solid surface affects the interactions significantly, i.e. the tight binding of proteins to carbohydrates on the array surface depends on multivalency of carbohydrates with appropriate spacing and orientation of carbohydrates on the surface. Recently, many glyco-macroligands have been used for glycan microarray fabrications, in particular with effort to control the density and orientation of the glycans on the microarray surface to thoroughly represent multi-dimensional cell surface carbohydrates closely. These novel approaches to control multivalency, density and the orientation of carbohydrates on the microarray surfaces have shown great potential for biomedical research and applications. This review highlights these recent developments of glyco-macroligand-based microarrays, primarily focusing on the fabrication of novel multi-dimensional glycan arrays with glyco-macroligands such as glycodendrimers, glycopolymers, glycoliposomes, neoglycoproteins, and glyconanoparticles and their potential biomedical applications as well (Fig. 1). In particular, how the multivalency, density and orientation of these glycans were controlled on the microarray surface and their impact on ligand binding to the carbohydrates presentation are discussed.
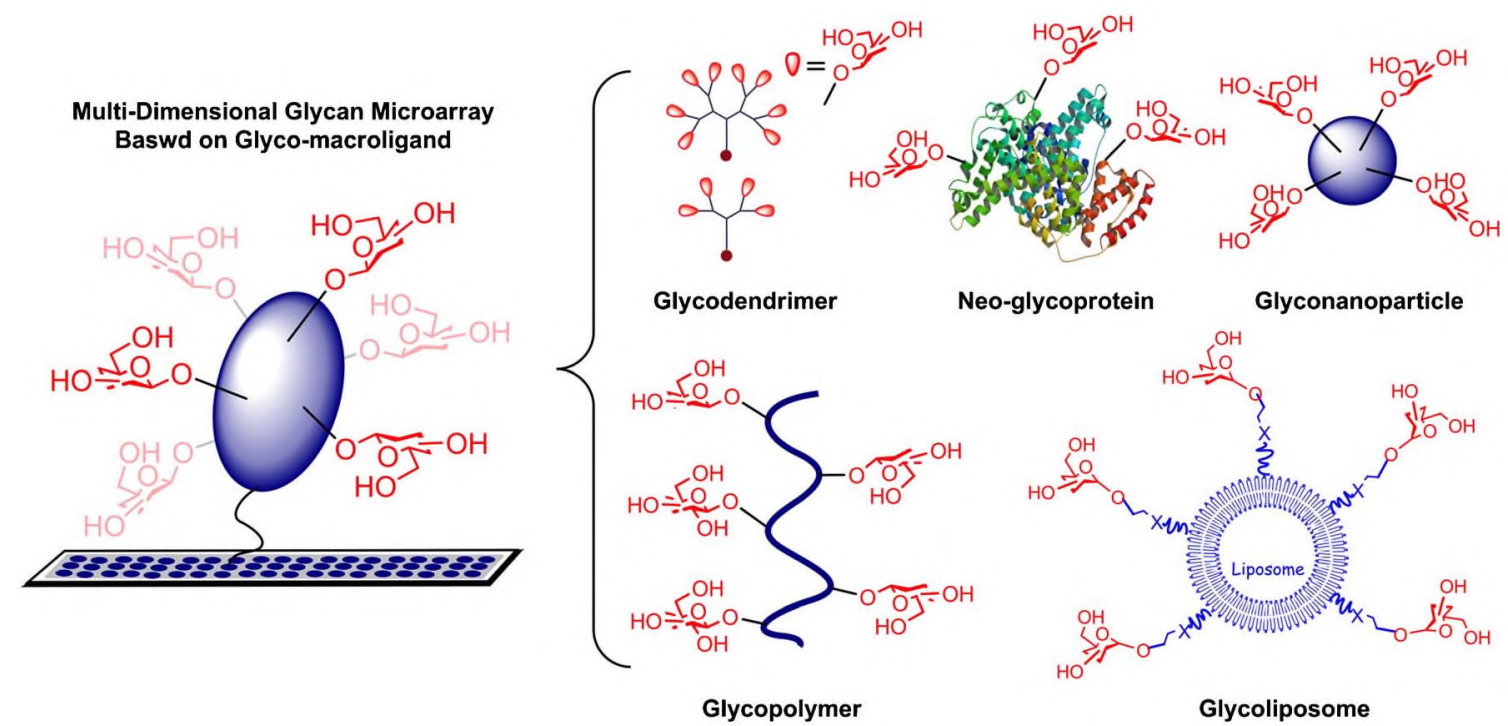

Fig. 1 Multi-dimensional glycan arrays with glyco-macroligands such as glycodendrimers, neo-glycoproteins, glycopolymers, glycoliposomes, and glyconanoparticles 


\section{Glycodendrimer-based glycan microarray}

Dendrimers are a class of highly branched, monodisperse globular macromolecules [16-18, 35, 36], which are characterized as highly branched 'dendrons' or 'wedges' that originate from one central multifunctional core unit and terminate in many functional groups around their peripheries [16]. Several research groups have confirmed the potential to use dendrimers as scaffolds for multivalent carbohydrate ligands by synthesizing carbohydrate containing dendrimers often termed as glycodendrimers $[17,18]$. Recently, the distinguishing multivalency of these glycodendrimers attained attention in synthesis of glycodendrimers for microarray application [19-22].

Pieters and coworkers reported glycodendrimer-based glycan microarray and studied the binding patterns of lectins to carbohydrates with respect to the multivalency of carbohydrate moieties and the inter-binding-site distances of lectins $[19,20]$. They designed mannose-based glycodendrimers and tested against two different lectins Con A and WGA [19], later, extended their work with five different glycodendrimers to evaluate a series of lectins [20]. Briefly, they synthesized alkyne-functionalized dendrimers with an amine functional group linked to the core which helps in immobilization of the dendrimers on solid surfaces. Azide functionalized sugars (Man, Gle, GleNAc, Gal, and Gal $\alpha 1,4 \mathrm{Gal}$ ), were introduced at the periphery by copper-catalyzed click chemistry followed by deprotection of sugars to afford glycodendrimers ranging from monovalent to octavalent dendrimers. These glycodendrimers were spotted on malemide-functionalized porous aluminum oxide surface (Fig. 2A) and binding affinities of several FITC-lectins (Con A, GNA, WGA, GS-1, LCA, PSA, DSA, PNA, CTB5, and LecA) were studied by flowthrough microarray technology. They tested the binding affinity of lectins Con A and GNA to mannose-based glycodendrimers microarray with varying concentrations ranging from 0.1 to $5 \mathrm{mM}$ and valences of mono, di-, tetraand octa-dendrimers. It was observed that the lectin Con A showed no major multivalency effect supporting the fact that, Con $\mathrm{A}$ is a tetramer with binding sites that are separated by over $60 \AA$ and are too far apart to binding over dendrimers.
However, lectin GNA with 12 binding sites spaced with a distance of $20 \AA$ showed significant increase in the binding to mannose dendrimers with increase in the valence of dendrimers from mono to octa. In their further studies [20], they investigated the multivalency effect on both binding affinity and specificity towards lectins. Lectin WGA with two closely spaced binding sites showed strong multivalent effect; also the binding was observed only to GlcNAcglycodendrimer at high valence and not to other glycodendrimers. The same phenomenon was observed with the other eight lectins that were tested against five different glycodendrimers, lectins with increase in inter-binding-site distance displayed decrease in the multivalency effect.

Wang and coworkers employed an altered approach for construction of glycodendrimer-based microarray by initially preparing a three dimensional dendrimeric platform followed by attaching the carbohydrate to the dendrimer surface [21]. Briefly, the poly(amido amine) (PAMAM) dendrimers immobilized glass slides were functionalized with hydrazide and aminoxy functional groups, followed by microspotting the carbohydrates dissolved in different buffers under microwave radiation energy (Fig. 2B). The PAMAM dendrimers tested in this study included five generations G1, G2, G3, G4 and G5 containing 8, 16, 32, 64 and 128 surface groups, respectively. For the detection of carbohydrate-lectin interactions, direct immunoassay was performed with individual or mixed Cy3-labeled lectins and sandwich immunoassay was performed with individual or biotinylated lectins and Cy3streptavidin. The cluster effect was tested by spotting oligosaccharides GlcNAc $\beta 1-4 G l c N A c$, GalNAc and GlcNAc onto aminoxy and hydrazide functionalized PAMAM dendrimers of all five generation, which were further incubated with Cy3labeled WGA. As a result, with the increase in the surface groups the fluorescent intensity increased, indicating the multivalency effect. Also, hydrazide-dendrimer coated slides provided higher fluorescent signal/noise ratio compared to aminoxy-dendrimers coated slides. Further, the specificity of different glyco-epitopes was examined through a panel of mono-, oligo- and polysaccharides by micro spotting them onto hydrazide dendrimers surface. These carbohydrate microarrays were incubated with either a mixture of biotin-
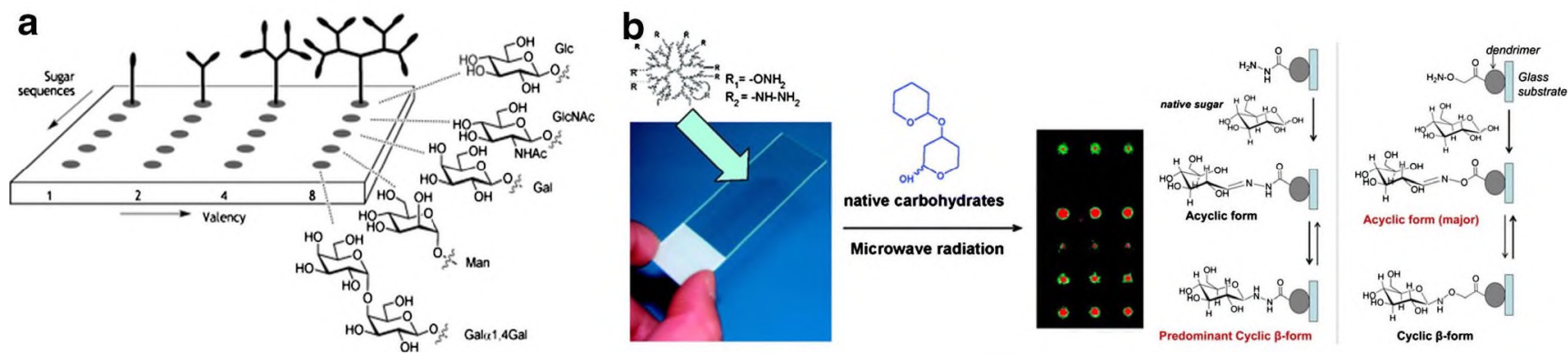

Fig. 2 Glycodendrimer-based glycan arrays: A Immobilized glycodendrimers via click chemistry [20] and B immobilization of oligosaccharides onto aminoxy-dendrimer and hydrazide-dendrimer coated glass slides [21]. Modified from Ref. [20, 21] 
labeled lectins and anti-sialyl lewis $\mathrm{x}$ antibody or with individual lectin/antibody separately and finally with Cy3streptavidin. The binding profiles verified the specificity of the carbohydrate epitopes on the hydrazide-dendrimer surfaces.

Miura and co-workers studied glycodendrimer microarray with surface Plasmon resonance (SPR) (20) and found that the binding kinetic constants were unique to each lectin, implying specificity of the multivalent effect of glycan-lectin interaction. The glycodendrimers with various generations and saccharides ( $\alpha$-Man, $\beta$-GIcNAc and $\beta$-Gal) were synthesized and immobilized on an acetylenyl-terminated gold substrate via click chemistry. The binding abilities of the saccharideimmobilized substrates were evaluated by SPR with the lectins of Con A, WGA and $\mathrm{RCA}_{120}$. Lectin binding to the glycodendrimer array was quantitatively analyzed, and association constants $\left(\mathrm{K}_{\mathrm{A}}\right)$ and kinetic rate constants $\left(\mathrm{k}_{\mathrm{a}}\right.$ and $\left.\mathrm{k}_{\mathrm{d}}\right)$ were evaluated.

Overall, glycodendrimers were successfully applied for microarray fabrication and were qualified in the screening of carbohydrate-protein interaction applications. These glycodendrimer microarrays were used not only to explore the protein-carbohydrate interactions but also assist in understanding the influence of multivalent carbohydrate display on the microarray surface in improving the sensitivity and selectivity of glycan microarrays. From the reported glycodendrimers microarrays it is recognized that the multivalency of the carbohydrates is well controllable by dendrimers. However, the spacing and orientation of the carbohydrates on the array surface are not yet structured by dendrimers to study their contribution to sensitivity and selectivity of glycan microarrays in capturing glycan binding molecules. This is a major drawback of the current glycodendrimers microarray techniques and continued studies on glycodendrimer design and its immobilization chemistry are further needed.

\section{Neo-glycoprotein-based glycan microarray}

Neo-glycoproteins are a group of glycomacroligands synthesized by covalently attaching glycans to proteins and show great potential as glycoprotein mimetics for biomedical research and applications [36, 37]. Neo-glycoproteins have also been applied for glycan microarray fabrication and have shown great potential for biomedical research and applications. Gildersleeve et al. developed neo-glycoproteins by covalently attaching carbohydrate moieties to lysine residues of bovine serum albumin (BSA) for microarray applications (Fig. 3) [11-13]. The carrier protein BSA served as both the multivalent scaffold and the linker for immobilization on to glass slides. Carbohydrates were conjugated to free amine groups of BSA via reductive amination of oligosaccharides or by coupling the oligosaccharides with carboxylic acid via activation with EDC/NHS. To test the glycan density effect, they synthesized 45 BSA-glycoconjugates by varying carbohydrate density of 11 different glycans on BSA [11]. These neo-glycoproteins were immobilized onto epoxy functionalized glass slides to test their binding patterns of plant lectins V. villosa $\mathrm{B}_{4}$ (VVL- $\left.\mathrm{B}_{4}\right), H$. Pomatia aggutinin (HBA), and soybean agglutinin (SBA) (Fig. 2A). As a result, they observed that the selectivity of lectins to a particular carbohydrate over other carbohydrates declined at high density and also the selectivity of conjugate for an individual lectin over other lectins declined at high density. Further, they also evaluated the density-dependent binding abilities of a set of monoclonal antibodies and determined that antibodies to the same carbohydrate antigen can recognize density in significantly different ways. Also, they investigated the density-dependent binding properties of serum antibodies from 30 subjects, their results demonstrated that modulation of glycan antigen density could be used to reveal differences in antibody populations between different subjects. The results from the above study revealed that both structure and the density of the carbohydrates significantly affect the affinity and selectivity to carbohydrate binding molecules.

Followed by this work, Gildersleeve group further developed an approach to modulate the neoglycoprotein density on the array surface [13]. Briefly, the glycan microarrays were constructed with approximately 600 combinations of glycans and presentations, with variations in density of neoglycoprotein, structure of glycans and the density of glycans. The microarray was designed to distinguish between the carbohydrate binding proteins that can form one-to-one complex (Fig. 3Ba), i.e. form a multivalent complex with a single neoglycoprotein or one protein binding to two or more neoglycoproteins, i.e. recognize carbohydrates on adjacent neoglycoproteins to form a bridge complex (Fig. 3Bb) and to study the effect of these bridging complexes with the neoglycoproteins spaced further apart on the surface (Fig. 2Bc). Their strategy to achieve this kind of array is to add unmodified protein (BSA) to the solution of neoglycoprotein and print these mixtures on the array surface, in which the unmodified BSA occupies surface between the neoglycoproteins based on the amount of unmodified BSA added to the mixture (Fig. 3Bc). They prepared the array surfaces with four different ratios of BSA per array component: 1:0 (100\% BSA), 1:1 (50\% BSA), 1:3 (25\% BSA) and 1:7 (12.5\% BSA), with 147 different neoglycoproteins and glycoproteins along with several controls to a total of 591 combinations. Plant lectins concanacalin A (Con A), Viciavillosaiso lectin B4 (VVL-B4) and Riciniscommunis agglutinin $\left(\mathrm{RCA}_{120}\right)$ were tested over the microarrays and observed that the spacing between the neoglycoproteins showed significant influence on the binding affinity of lectins. 
Fig. 3 Neo-glycoprotein-based glycoarray: A neoglycoprotein with variations in carbohydrates and density on the array surface [11], B multivalent binding modes and the array strategy of neoglycoprotein [13], C illustration of high and low density neoglycoprotein on the surface [33]. Modified from Ref. $[11,13,33]$

a

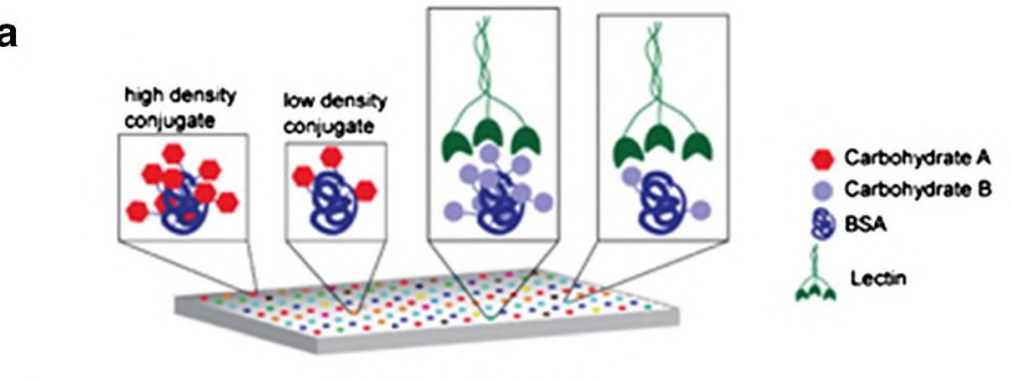

b

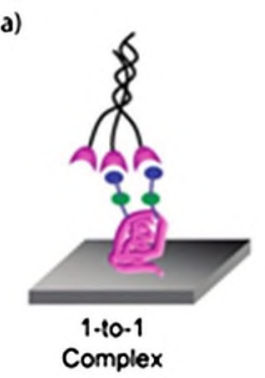

Carbohydrate microarray b)

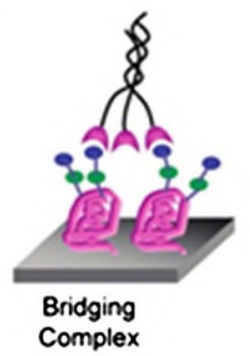

c)

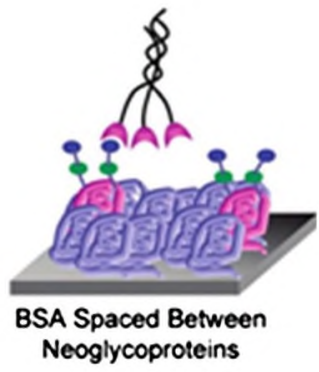

Legend

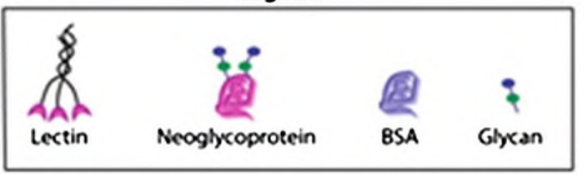

C

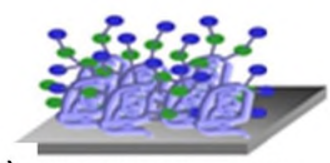

a)

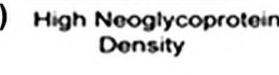

b) Low Nooglycoprotein

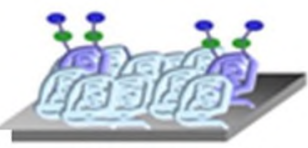

Legend

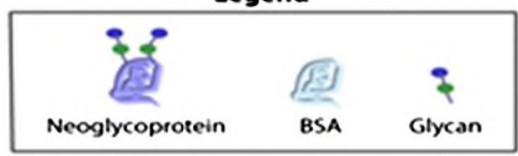

Later on, the same group studied the effects of neoglycoprotein density on antibody binding [33]. Briefly, they investigated the binding affinities of five commercially available antibodies 81FR2.2, HE-195, HE-193, B480, and $\mathrm{Z} 2 \mathrm{~A}$ to several antigens and found that neoglycoprotein density had a considerable effect on antibody recognition (Fig. 3C). Further, the binding affinities of antibodies from human serum from 15 healthy subjects were analyzed and determined that the binding affinities of different subpopulation were different based on the density of neoglycoprotein. In addition, they evaluated the immune response induced by prostate cancer vaccine from six individuals before and after vaccination and verified that the variations in neoglycoprotein density could be used to detect antibody responses. Overall, these researches demonstrated that the density of the carbohydrates on the array surface can be adjusted by using neoglycoproteins with different carbohydrate density on it, which are significant in studying the carbohydrate binding ligands. These studies set up a general protocol glycan microarray techniques using any kind of carbohydrates and screening their functions and potential biomedical applications.

\section{Glycopolymer-based glycan microarray}

Glycopolymers carrying pendant sugar moieties on the polymer backbone as multivalent carbohydrate derivatives have been proven as an effective tool to study carbohydrate-based biological processes and have shown great potential in biomedical research and applications [38]. Glycopolymers are 
a

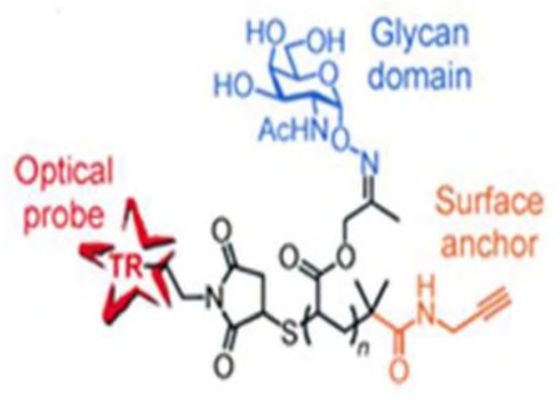

b CONVENTIONAL ARRAY

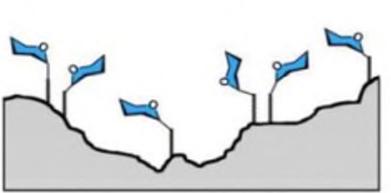

poorly controlled glycan presentation
MUCIN MIMETIC ARRAY

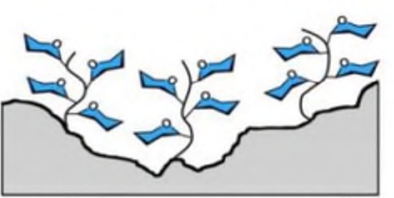

polymer structure defines glycan display

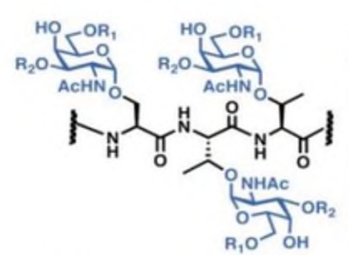

$\mathbf{R}_{1}, \mathbf{R}_{2}=$ oligosaccharides native mucin

d

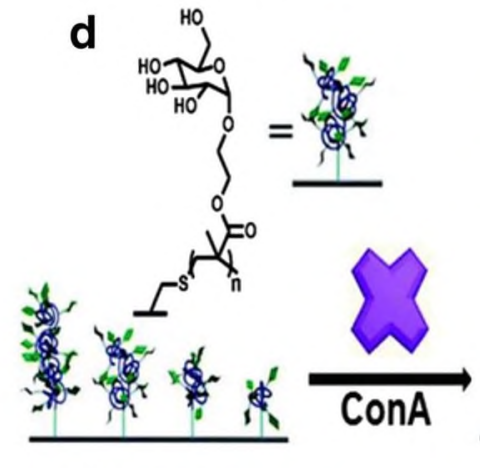

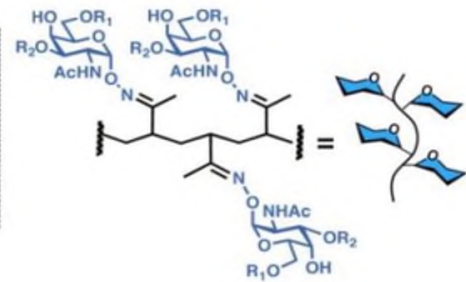

mucin mimetic

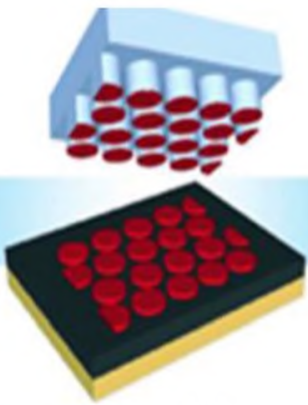

C
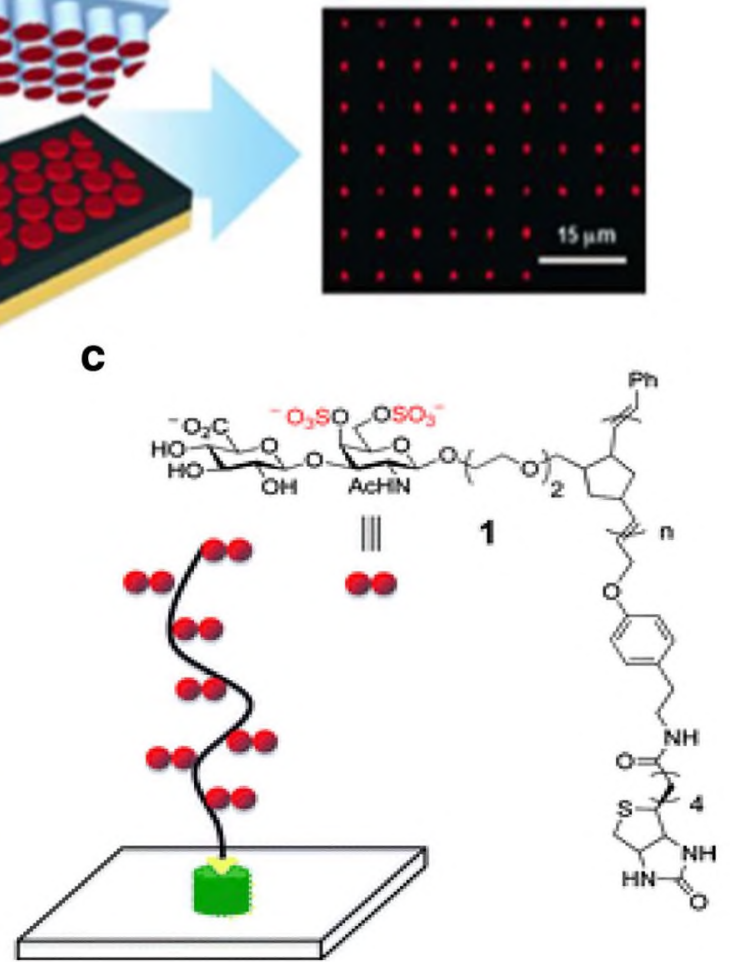

e
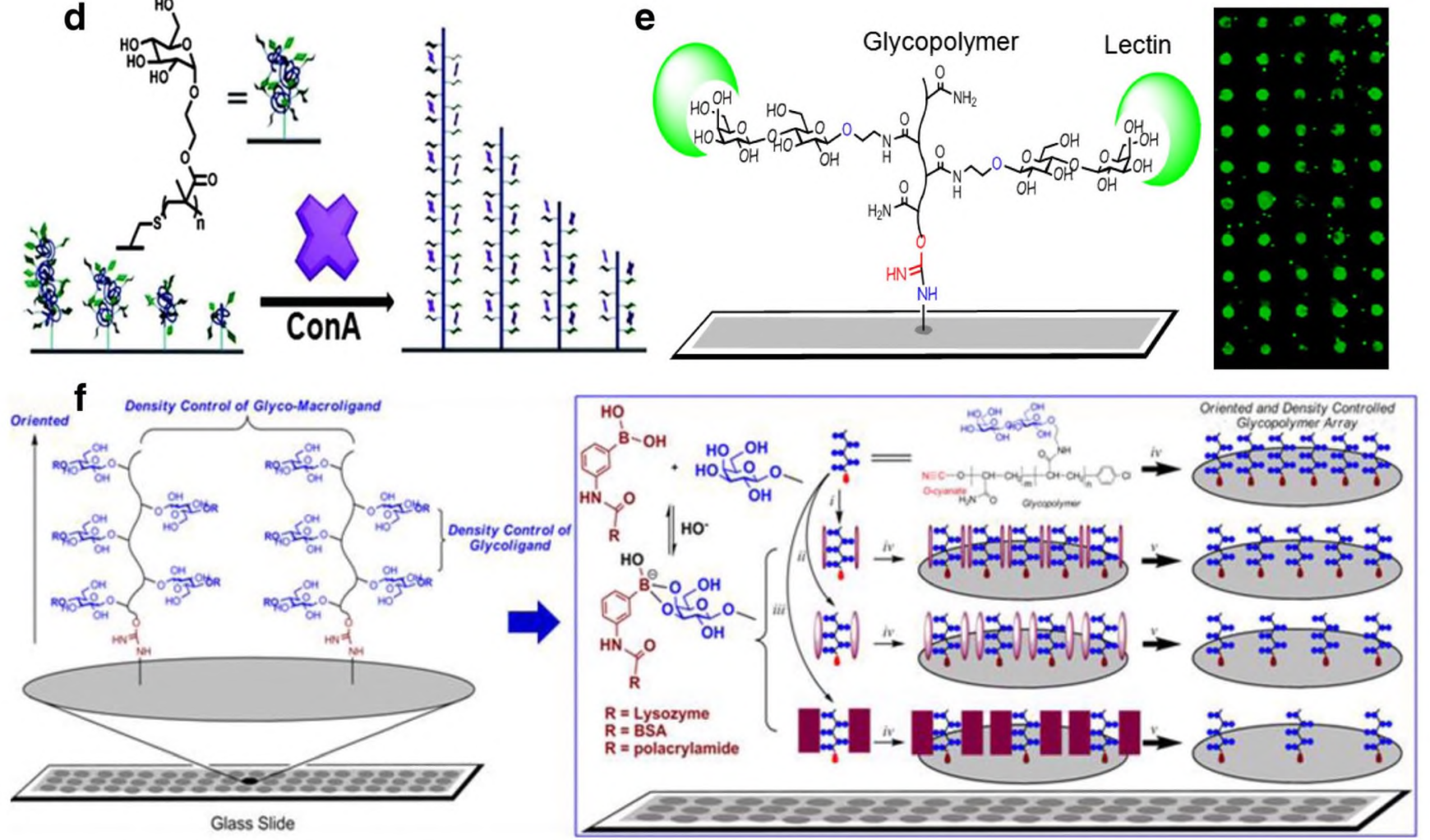

Fig. 4 Glycopolymer-based glycoarray: a duel end functionalized mucin like glycopolymer with alkyne on one end and Texas Red dye on the other end [24], b mucin like glycopolymer presentation that mimics native mucin [40], c biotin end functionalized glycopolymer immobilized glass slide [26], d glycopolymer formation at different illumination times [41], e O-cyanate end functionalized glycopolymer immobilized on amine functionalized glass slide [27], f O-cyanate chain-end functionalized glycopolymer pre-complexed and immobilized with boronic acid ligands of different sizes [28]. Modified from Ref. [24, $26-28,40,411$ 
either synthesized by direct polymerization of carbohydratecontaining monomers with or with out protection group or by the post polymerization glyco-conjugation of synthetic polymers [23]. It is generally accepted that synthetic glycopolymers can mimic the functions of naturally occurring polysaccharides $[28,29,38]$ and have been employed as binding cell-surface receptors $[30,38]$. The strength and selectivity of binding interactions between multivalently displayed carbohydrates and targets are likely to depend on the density and relative spatial arrangement of the carbohydrate residues of glycopolymers. In recent years, chain-end functionalized glycopolymers were synthesized and demonstrated homogeneous immobilization of the glycopolymer via a single terminal anchor to yield an oriented and density controlled exhibition of multivalent pendant carbohydrates and were used for glycan microarray applications [38].

Bertozzi's group synthesized a duel end functionalized mucin like glycopolymer [24] with a center mucin mimetic domain with $N$-acetylgalactosamine moieties, one terminal alkyne group as surface attachment element and the other terminal outfitted for conjugation of Texas Red as a fluorophore. By microcontact printing the alkyne terminal glycopolymers were immobilized onto azide functionalized surface in presence of a copper catalyst, which forms a stable triazol linkage to covalently immobilize the glycopolymer. As the glycopolymer was also functionalized with fluorescent tag Texas Red on the other end the immobilization of polymer was confirmed by fluorescent microscopy. Also, the ligand specificity of glycans to the polymer was tested by immobilizing nonfluorescent tag glycopolymer with Texas Red tagged Helixpomatia agglutinin (Texas Red-HPA) that recognizes $\alpha$-GalNAc. Examination of the surface by fluorescent microscopy revealed the specific binding of HPA only to $\alpha$-GalNAc (Fig. 4a) confirming the specificity of glycans. They later developed an approach for the synthesis of glycopolymers for microarray applications via ligation of reducing sugars to the polymer backbones carrying hydrazide groups and end functional biotin group [25]. They arrayed a cluster of biotinylated glycopolymers onto streptavidin-coated glass slides, which were recognized by lectins specifically depending on their pendant glycans.

Bertozzi et al. also synthesized duel end functionalized mucin like glycopolymers with $\alpha-N$-acetylgalactosamine $(\alpha$ GalNAc) moieties via RAFT polymerization for studying the cross-linking of mucin like glycoconjugates by lectins [39]. The polymer was terminated with biotin on one end to facilitate anchoring of glycopolymer on to streptavidin-coated microarray substrates and a trithiocarbonate moiety on the other end that provided a free sulfhydryl group for conjugation of a maleimide-functionalized Cy3 dye upon rapid aminolysis with cysteamine in DMF. They synthesized five mucin-like glycopolymers with a range of GalNAc valencies $(68,92,111$, 146, and 170). To control the density of the glycopolymer on the surface they spotted glycopolymer solutions of different concentrations $(400,150$, and $75 \mathrm{nM})$ on the streptavidin coated glass slides. Apparent dissociation constants of four lectins (Soybean agglutinin (SBA), Wisteria floribunda lectin (WFL), Vicia villosa-B-4 agglutinin (VVA), and Helix pomatiaagglutin (HPA)) with specificity to $\alpha$-GalNAc were determined. They observed valency dependent binding for SBA, WFL and VVA lectins, whereas HPA showed strong avidities toward all the polymers irrespective of GalNAc valency. Though all four lectins have capacity to cross-link low valency glycoconjugates, only the high valency GalNAc mucin mimetic polymer showed cross-linking to SBA, which reveals that glycan valency and organization are critical parameters to determine the cross-linking properties of lectins (Fig. 4b).

Glycosaminoglycans play important roles on cell surface. Hsieh-Wilson et al. synthesized a biotin end functionalized glycopolymer that mimic chondroitin sulfate proteoglycans via ring-opening metathesis polymerization technique [26], which were employed for microarray and SPR applications. Both sulfated CS-E and non-sulfated CS-C glycopolymers were immobilized onto streptavidin coated glass slides by high-precision contact-printing robot, which delivers nanoliter volumes of glycopolymer onto glass slides. Polymer immobilized glass slides (Fig. 4c) were tested for binding of specific monoclonal antibodies 2D11 and 2D5, which are selective for CS-E and CS-C glycopolymers, respectively. They also studied the binding of a growth factor, glial cell-derived neurotropic factors selective for sulfated epitopes, which showed higher selectivity for CS-E compared to CS-C by both microarray and SPR techniques.

Most recently, Braunschweig et al. employed a new approach by combining polymer pen lithiography (PPL) and beam pen lithiography (BPL) with acrylate or methacrylate photo polymerization chemistry to generate arrays of brush polymers with side chain functionalized with fluorophores or glycans [40]. They patterned acrylate and methacrylate monomers ( $\alpha$-mannose and $\alpha$-glucoside) onto thiol coated glass slides and exposed these glass slides to UV light that produced brush polymers by a photoinduced radical acrylate polymerization reaction. Both the monolayers and the brush polymers were treated with fluorophore labeled Con A at different concentrations, to investigate the architecture effect of these glycans in binding to Con A. The fluorescence intensity for the brush polymers binding was observed to be 20 times greater than that for glycan monolayers at high concentrations of Con A. The different polymer brush heights were attained by variation in irradiation times $(2,5,10$ and $20 \mathrm{~min})$, with $126 \mathrm{~nm}$ height polymers at $20 \mathrm{~min}$ whereas $8 \mathrm{~nm}$ height polymers at $2 \mathrm{~min}$, which was determined by AFM. Also, they 
found that the polymers with longer length $(126 \mathrm{~nm})$ are an order of magnitude more sensitive than shorter polymers ( $8 \mathrm{~nm}$ ) towards binding Con A (Fig. $4 \mathrm{~d}$ ).

We recently developed an approach for glycan microarrays using glycopolymer to control orientation and density with $O$-cyanate chain-end functionalized glycopolymers $[27,28]$. Initially, we synthesized an $O$-cyanate chain end functionalized glycopolymer with pendant lactose moieties on the backbone via cynaoxyl-mediated free-radical polymerization technique [27]. In our study, glycopolymer microarray (spot size $500 \mu \mathrm{m}$ diameter) was fabricated by micro contact stamping glycopolymer onto amine functionalized glass slide and incubated the glass slides with lectin-FITC (Arachishypogae, FITC-Labeled, Sigma), then subjected them to fluorescent imaging. A control was prepared by incubating the glycopolymer array with lactose pre-incubated lectin-FITC instead of free lectin. Fluorescence microscopy analysis of the surfaces revealed specific binding of lectin to the immobilized glycopolymer (Fig. 4e). The lectin binding to arrayed glycopolymer was inhibited in the presence of free lactose, further confirming the specific lectin binding to the glycopolymer. Moreover, glycopolymer array with different concentration of glycopolymer showed concentration-dependent lectin binding to glycopolymer.

The glycopoymer-based microarrays showed great potentials as multi-dimensional glycan microarrays. However, the density of the immobilized glycopolymer on the array surface is still uncontrollable, and thus, the possibility to access the multivalent glycans in parallel maybe limited and therefore does not facilitate maximum protein binding affinity and specificity. We proposed a novel approach to control the density of glycopolymers on the array surface to solve this problem [28]. First, we examined the glycan density effect for lectin binding in the glycopolymer by synthesizing a series of glycopolymers with different densities of pendant glycan and molecular weights. Four kinds of glycopolymer were synthesized and glycopolymer microarrays were fabricated by microcontact stamping of glycopolymers onto amine functionalized glass slides followed by incubating with PNA lectin-FITC. Glycopolymer with 1 to 30 ratio of lactose and acryl amide showed the highest level of lectin binding compared to the glycopolymers with 1 to 18,1 to 54 and 1 to 51 ratio of lactose and acryl amide. These results indicated that the glycan density in the polymer has impact for the lectin binding. Next, for controlling the density of immobilized glycopolymer on array surface; we developed end-point immobilization of glycopolymer combined with molecular spacing technique. Briefly, $O$-cyanate chain-end functionalized glycopolymer was pre-complexed with boronic acid ligands in different sizes and then immobilized onto aminefunctionalized glass slides. After the immobilization, the spacer boronic acid ligands were released from the immobilized glycopolymers at reduced $\mathrm{pH}$ condition to afford the oriented and density controlled glycopolymer microarray (Fig. 4f). We synthesized three macro-boronic acid moieties lysozyme-BA (MW: about $15 \mathrm{kDa}$ ), BSABA (MW: about $70 \mathrm{kDa}$ ) and polyacrylamide-BA (MW: about $10 \mathrm{kDa}$ ) as a spacing molecule to vary immobilized glycopolymer density. These glass slides were then incubated with two different lectins PNA and RCAI, both have specificity to $\beta$-galactose. Interestingly, the binding of both lectins was enhanced when the spacers were used for immobilization. PNA and RCAI showed higher binding for different ratios of glycopolymer to BA-spacer, stating that lectins that bind same glycans are affected in unique way. Further, this density-dependent lectin binding specificity was confirmed by SPR technique. Overall, these results indicated that oriented glycopolymers with controlled ligand density facilitates enhanced protein binding, which is very important for studying glycan-protein interaction, such as assessing multivalent glycans in parallel in a microchip format and other solid phase assays such as biosensors.

\section{Glyco-membrane mimetics-based glycan microarray}

Cell membrane mimetic systems play fundamental roles in understanding molecular interactions on the cell surface and provide enormous opportunities in developing products for biomedical research and applications [41]. Membrane mimetic systems can be designed to have controlled environment that mimic the characteristics of a native membrane, such as composition, structure, curvature and charge close to their native structure as in the cell membrane [42]. A variety of membrane mimetic systems have been developed for investigation of various membrane-related processes. Liposomes are one of the most common membrane mimetic systems, which are small artificial vesicles of spherical shape that can be created from cholesterol and natural non-toxic phospholipids [43, 44]. Lipid composition, surface charge, size, and the method of preparation are key factors that affect the liposome properties [45]. In addition, supported lipid membrane system emerged as the most attractive platform because of its quasi-natural setting and that its size, geometry and composition can be tailored with great precision to the complexity of biological membrane. Several platforms such as solid-supported [46-50], tethered [51-53], polymer cushioned [54-56] lipid bilayers and supported vesicular systems [57, 58] have been developed. So far, glyco-membrane mimetic systems such as supported glyco-lipid membrane systems and glyco-liposomes have been used for glycan microarray applications [29, 30, 59]. 


\section{Supported glyco-lipid bilayer-based glycan microarray}

Guo et al. demonstrated a fluidic glycan microarray for quantitative glycomics application [29] based on robust supported lipid bilayer (SLB) technology. This approach is to incorporate tethered cholesteryl groups onto the bottom leaflet of the supported bilayer lipid membrane to increase the stability and rigidity of the membrane, also combined with tether achieves desired robustness (Fig. 5). Briefly, a supported lipid bilayer was formed by spotting small unilamellar vesicle (SUV) onto the tethered cholesterol and verified the fluidity of SLB by fluorescence recovery after photo bleaching. Different concentrations of mannose linked lipids were incorporated in the lipid mixture to fabricate density gradient microarray with known mannose density of each spot. The mannose-based microarray was then incubated with a suspension of $E$. coli. ORN 178 and ORN208 as a negative control. It was found that high $E$. coli. adhesion was observed with the increase of mannose density. The number of cells adhered to the array slide was counted, the fluorescent microscopy of $E$. coli. adhesion to the SLB sports containing $0.1,1,5$ and $10 \%$ mannose and there is a rapid increase in the cell count at $\geq 5 \%$ and reached saturation later on. Also, an inhibition study was conducted where the mannose nanoparticles were added to the suspension of $E$. coli. As a competitive inhibitor, and with increase in the concentration of nanoparticles the cell adhesion to the mannose array decreased. This study indicated that the dynamic clustering of mannosyl groups on the fluidic membrane surface could simulate the function of complex oligo- and high-mannose molecules.

\section{Glyco-liposome-based glycan microarray}

Liposome microarrays have been investigated for several applications in membrane biophysics, biotechnology, and colloid and interface science $[57,58]$. We recently investigated the application of azide-reactive liposome for efficient and chemically selective immobilization and microarray fabrication (Fig. 6a) [30]. Azide reactive liposomes were synthesized with DSPE-PEG 2000 -triphenylphosphine, phospholipids (DSPE) and cholesterol rapid extrusion method. Azide$\mathrm{PEG}_{6}$-Biotin was conjugated to prepared liposomes in 7.4 $\mathrm{pH}$ PBS buffer via Staudinger ligation and immobilization of these biotinylated liposomes was performed by incubating them onto streptavidin functionalized glass slides. To confirm the immobilization of intact liposomes on glass slides, DSPE rhodamine dye was doped into the lipid bilayer into both post biotinylated and directly biotinylated liposomes and the 5,6carboxyfluorescene dye was encapsulated inside the liposomes and subjected to fluorescence imaging. Both rhodamine and the 5,6-CF tags were observed at respective wavelengths confirming that the arrayed intact liposomes were achieved through specific biotin/streptavidin interaction. Next, the azide reactive liposomes were immobilized on azide functionalized glass slides and later subjected to glycosylation by conjugation of 2-azideethyl-lactose to leftover TP on the liposome's exterior surface. Lectin binding studies were conducted by incubating the liposome immobilized glass slide with lectin PNA. The specific lectin binding to lactosylated liposome was observed, while no lectin binding was observed to liposome with just anchor group.
Fig. 5 Formation of a glycan presenting supported lipid bilayer (SLB) surface from a small unilamellar vesicle (SUV) solution and glycan density gradient microarray for pathogen adhesion [29]
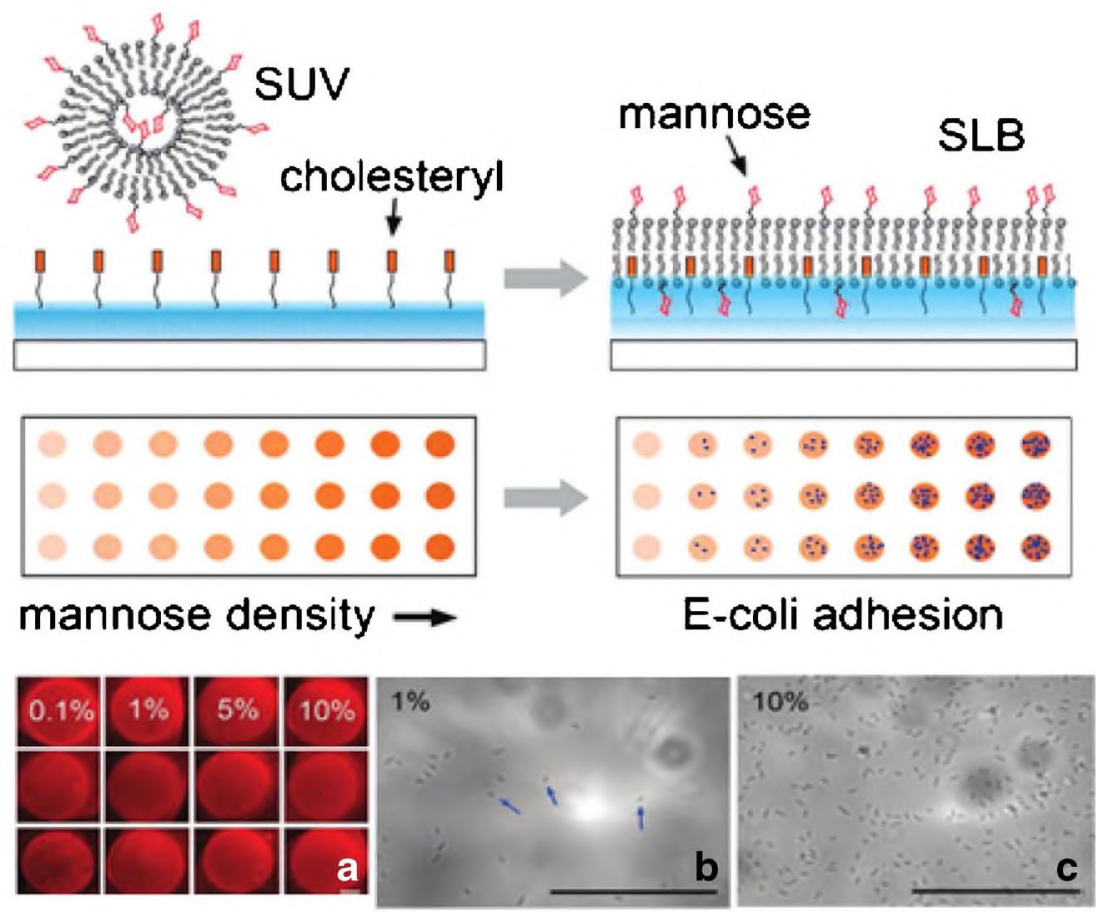


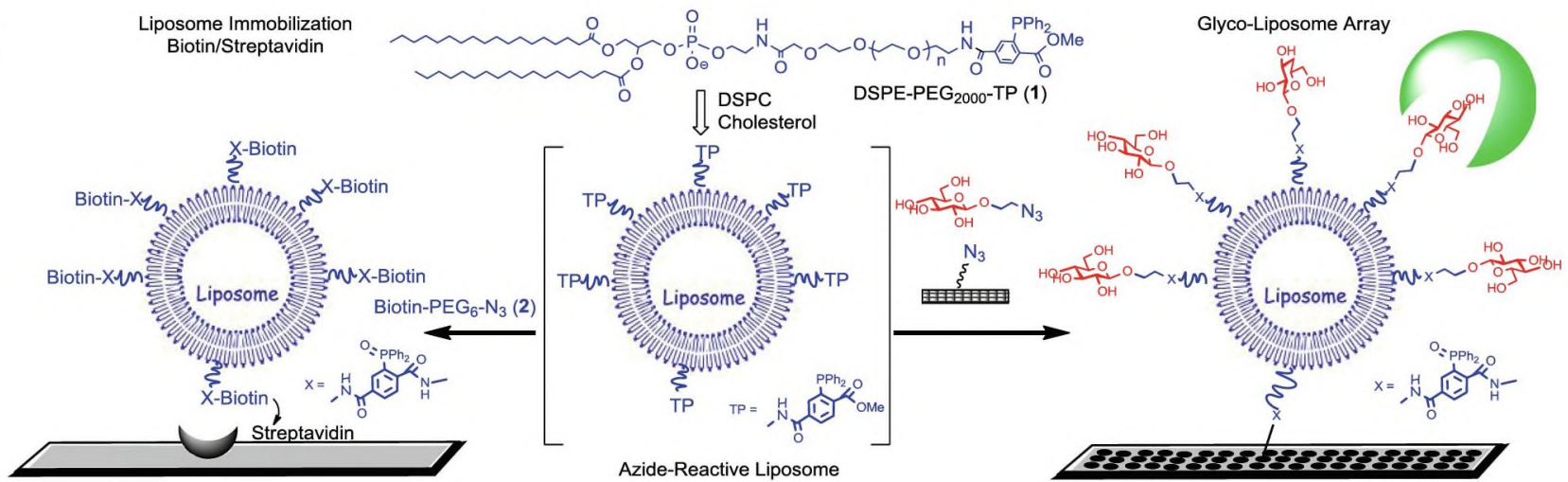

b
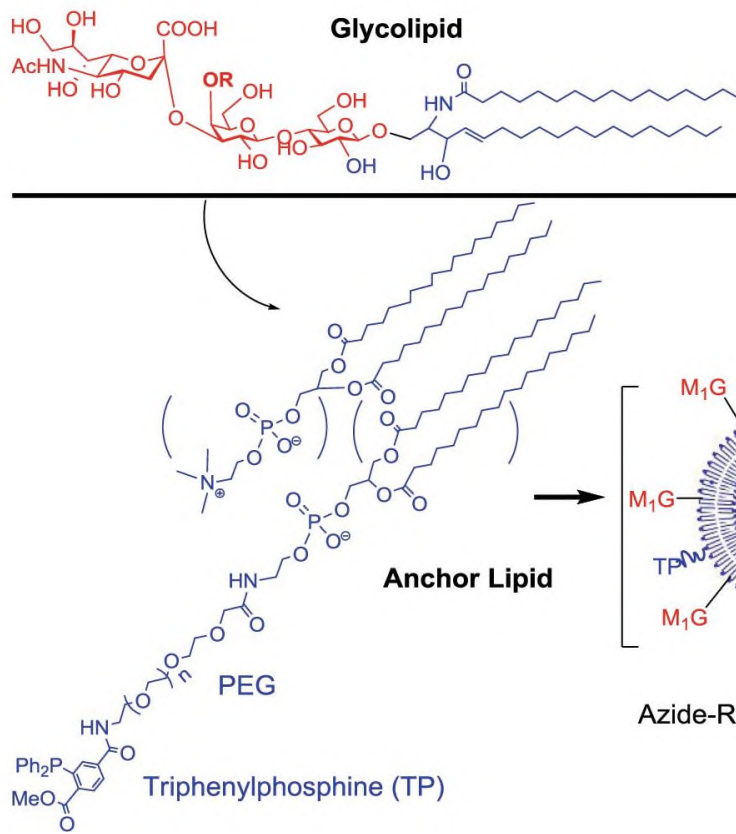

Liposomal Glyco-Microarray

Ganglioside

$\mathrm{GM}_{1}, \mathrm{R}=$ Lactosamine, $\mathrm{GM}_{3}, \mathrm{R}=\mathrm{H}$

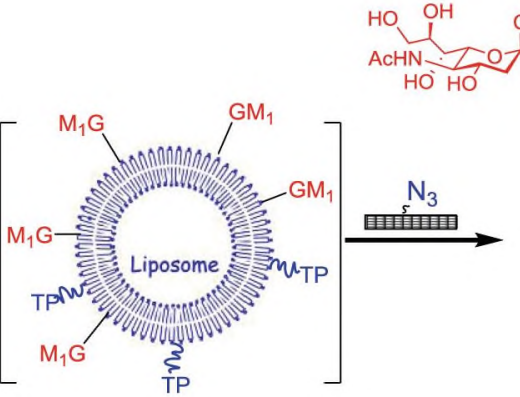

Azide-Reactive Liposome $\mathrm{COOH}$

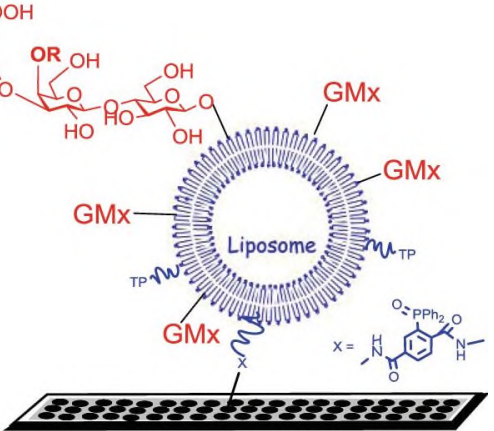

Fig. 6 Glyco-liposome-based glycoarray: a Surface functionalization, immobilization and glyco-functionalization of liposome via Staudinger ligation [30], b liposomal glyco-microarray formation based immobilization via Staudinger ligation [59]. Modified from Ref. [30, 59]

Further, this work was extended to study the glycolipid and protein interactions [59] by incorporating natural and synthetic glycolipids into liposomes, which were immobilized onto an azide functionalized glass slide for microarray application (Fig. 6b). Briefly, liposomes with DDPE-PEG $2000-\mathrm{TP}$ and gangliosides were prepared and printed on azide functionalized glass slide to study the interactions between glycolipids and lectins. These microarrays mimic the presentation of carbohydrates on the cell surface. The lipid bilayer microenvironment impacts the modulation of the glycolipid presentation and the fluidity of the lipid bilayer allows optimal geometric positioning of the glycan head groups. In our study, the intact liposomes with natural glycolipids GM1 and GM3 were printed on an azide functionalized glass slide; liposomal GM1 was immobilized at different range of concentrations from 10 to $400 \mu \mathrm{g} / \mathrm{mL}$ and incubated with FITC-cholera toxin B (CTB). Also, the GM1 liposomal microarray was incubated with different concentrations of CTB. The binding of CTB to GM1 liposome increased with increase in the concentration of GM1 as well as CTB but reached saturation at $60 \mu \mathrm{g} / \mathrm{mL}$ of CTB indicating that the binding is density dependent. Also, the GM1 microarray was incubated with lectins MAA and PNA, which binds specifically to $\alpha(2-3)$-sialic acid and $\beta$ galactose, respectively. The binding curve reached saturation at $100 \mu \mathrm{g} / \mathrm{mL}$ for both MAA and PNA. GM3 microarray was also incubated with MAA and observed that the binding reached saturation at $100 \mu \mathrm{g} / \mathrm{mL}$. These binding curves indicate that the liposomal microarrays with different glycolipids show specific density dependent binding capacity and affinity as well. The liposomal glyco-microarrays have advantages of providing mobility to glycans by closely mimicking the cell surface glycans and also controlling the surface glycan density in an immobilized state and thus provide an important tool to study cell surface carbohydrate functions. 


\section{Glyco-nanoparticle-based glycan array}

Nanoparticles have been used enormously for biomedical applications as the size of the nanoparticles is close to that of biomolecules [60]. Glyco-nanoparticles (GNPs), sugar-coated nanoparticles, were synthesized in the past decade to study the interactions of carbohydrate and carbohydrate binding molecules [61]. Three major types of nanoparticles functionalized with glycans have been reported so far: gold and silver glyconanoparticles, semiconductor glyco-quantum dots and magnetic glyconanoparticles.

Yan et al. recently reported glyco-nanoparticle (GNP)based microarrays [34], where glyco-nanoparticles were printed on a photoactive surface followed by covalent immobilization by light activation. Briefly, GNPs were synthesized and functionalized with perfluoropernylazide (PFPA) followed by conjugating five different carbohydrates to GNPs using photocoupling chemistry. Epoxy functionalized wafers were treated with PAAm-PFPA, then the GNPs were printed followed by coating the surface with polystyrene (PS) or poly(2-ethyl-2-oxazoline) (PEOX) to avoid nonspecific binding of lectins to the surface (Fig. 7). These glycan microarray surfaces were tested with lectin Con A labeled with fluorescein-doped silica nanoparticle (FSNP-Con A) or FITC labeled lectins (FITC-Con A) and it was observed that the FSNP-Con A showed enhanced signal then the FITC-Con A. GNP microarrays were compared with conventional glycan arrays by treating them with FSNP-Con A and observed higher signals for high affinity ligands Man3, Man2 on GNP microarray than in carbohydrate microarray. These results indicated that the GNP microarrays increase the affinity and selectivity when compared to carbohydrates alone on the array.

\section{Conclusion}

Overall, glycan microarrays have been widely employed to study the interactions between carbohydrates and carbohydrate binding molecules and show great potentials for biomedical research and applications. The glycan presentation on the array surface extremely affects the binding affinity and specificity of these glycan binding molecules. Several glyco-macroligands were developed to control the glycan presentation on the surface in terms of multivalency, density and orientation of glycan on the array surface. These glyco-macroligands microarray contributed greatly to understand the importance of presentation of glycans on the surface in terms of specificity and selectivity of glycan binding molecules. However, it is still difficult to control the presentation of glycans precisely for every glycan binding molecule on the surface as the requirement of the glycan density and orientation changes according to the type of glycan binding molecule of interest. The site-specific immobilization of glyco-macrolig and is still a challenge for controlling its orientation and density on the array surface as well. Therefore, there is a lot of scope for improvement in developing methods for varying glycan presentation and extending the applications of carbohydrate microarrays.
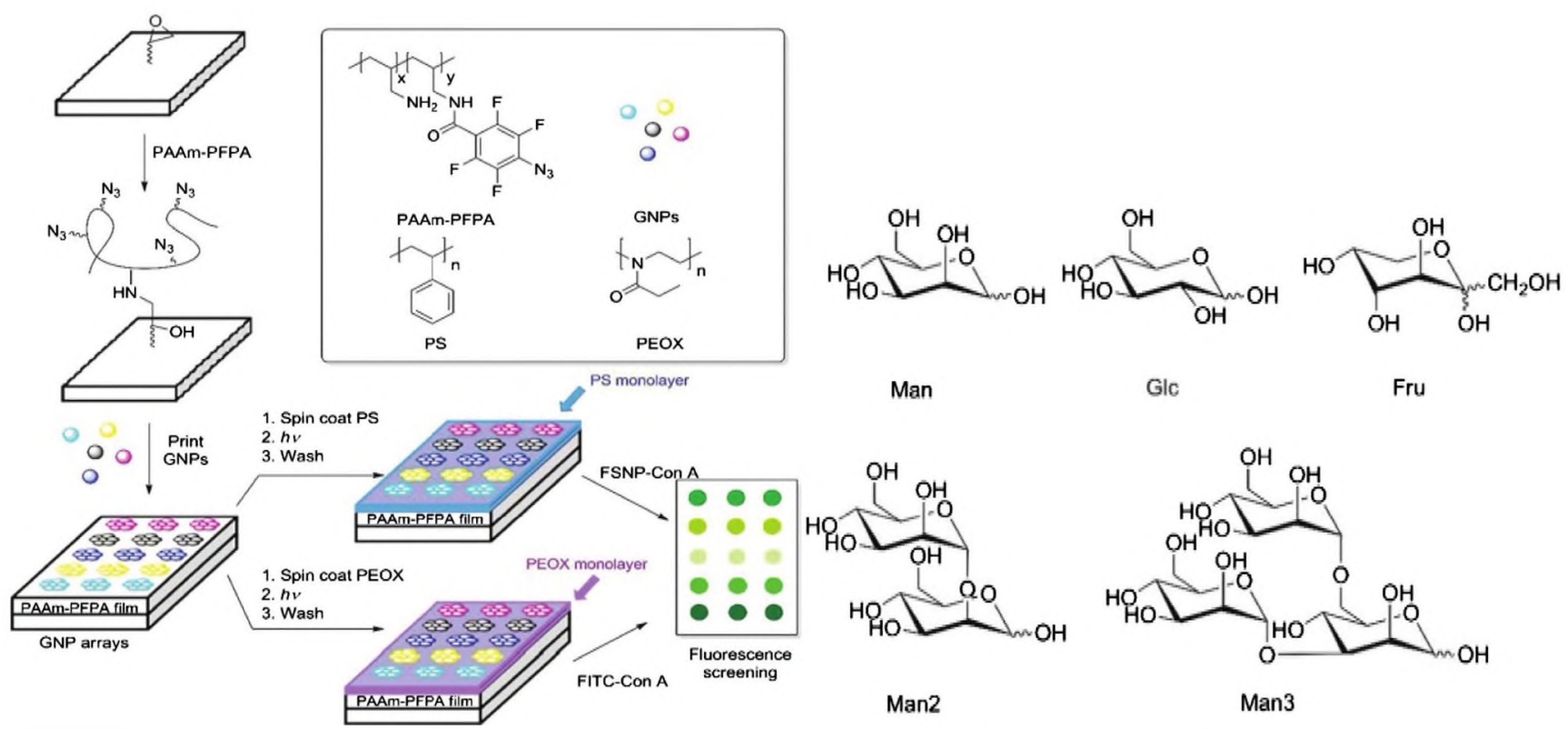

Fig. 7 Glyconanoparticle microarray with Man 3, Man 2, Man, Gle, Fru and interactions with FSNP-Con A or FITC-Con A [34] 
Acknowledgments This work was supported by American Heart Association Grant-in Aid (14GRANT20290002) and Faculty Research Development Fund and the research fund from the Center for Gene Regulation in Health and Disease (GRHD) at Cleveland State University supported by Ohio Department of Development (ODOD). This work was partially supported by grants from The National Natural Science Foundation of China (31328006). $\mathrm{H}$, Nie appreciates the China Oversea Scholar Award from China Scholarship Council

\section{References}

1. Lis, H., Sharon, N.: Lectins: carbohydrate-specific proteins that mediate cellular recognition. Chem. Rev. 98, 637-674 (1998)

2. Flitsch, S.L., Ulijn, R.V.: Sugars tied to the spot. Nature 421(6920), 219-220 (2003)

3. Sodetz, J.M., Paulson, J.C., McKee, P.A.: Carbohydrate composition and identification of blood group $\mathrm{A}, \mathrm{B}$, and $\mathrm{H}$ oligosaccharide structures on human Factor VIII/von Willebrand factor. J. Biol. Chem. 254, 10754-10760 (1979)

4. Karlsson, K.-A.: Microbial recognition of target-cell glycoconjugates. Curr. Opin. Struct. Biol. 5(5), 622-635 (1995)

5. Lee, K.J., Mao, S., Sun, C., Gao, C., Blixt, O., Arrues, S., Hom, L.G., Kaufmann, G.F., Hoffman, T.Z., Coyle, A.R., Paulson, J., Felding-Habermann, B., Janda, K.D.: Phage-display selection of a human single-chain Fv antibody highly specific for melanoma and breast cancer cells using a chemoenzymatically cynthesized GM3carbohydrate Antigen. J. Am. Chem. Soc. 124, 12439-12446 (2002)

6. Varki, A.: Biological roles of oligosaccharides: all of the theories are correct. Glycobiology 3(2), 97-130 (1993)

7. Park, S., Shin, I.: Fabrication of carbohydrate chips for studying protein-carbohydrate interactions. Angew. Chem. 114(17), 3312 3314 (2002)

8. Fukui, S., Feizi, T., Galustian, C., Lawson, A.M., Chai, W.: Oligosaccharide microarrays for high-throughput detection and specificity assignments of carbohydrate-protein interactions. Nat. Biotechnol. 20(10), 1011-1017 (2002)

9. Schwarz, M., Spector, L., Gargir, A., Shtevi, A., Gortler, M., Altstock, R.T., Dukler, A.A., Dotan, N.: A new kind of carbohydrate array, its use for profiling antiglycan antibodies, and the discovery of a novel human cellulose-binding antibody. Glycobiology 13(11), 749-754 (2003)

10. Disney, M.D., Seeberger, P.H.: The use of carbohydrate microarrays to study carbohydrate-cell interactions and to detect pathogens. Chem. Biol. 11(12), 1701-1707 (2004)

11. Oyelaran, O., Li, Q., Farnsworth, D., Gildersleeve, J.C.: Microarrays with varying carbohydrate density reveal distinct subpopulations of serum antibodies. J. Proteome Res. 8(7), 3529-3538 (2009)

12. Qiu, Y., Patwa, T.H., Xu, L., Shedden, K., Misek, D.E., Tuck, M., Jin, G., Ruffin, M.T., Turgeon, D.K., Synal, S., Bresalier, R., Marcon, N., Brenner, D.E., Lubman, D.M.: Plasma glycoprotein profiling for colorectal cancer biomarker identification by lectin glycoarray and lectin blot. J. Proteome Res. 7(4), 1693-1703 (2008)

13. Zhang, Y., Li, Q., Rodriguez, L.G., Gildersleeve, J.C.: An arraybased method to identify multivalent inhibitors. J. Am. Chem. Soc. 132(28), $9653-9662$ (2010)

14. Park, S., Gildersleeve, J.C., Blixt, O., Shin, I.: Carbohydrate microarrays. Chem. Soc. Rev. 42(10), 4310-4326 (2013)

15. Wang, L., Cummings, R.D., Smith, D.F., Huflejt, M., Campbell, C.T., Gildersleeve, J.C., Gerlach, J.Q., Kilcoyne, M., Joshi, L., Serna, S., Reichardt, N.C., Parera, P.N., Pieters, R.J., Eng, W.,
Mahal, L.K.: Cross-platform comparison of glycan microarray formats. Glycobiology 24(6), 507-517 (2014)

16. Turnbull, W.B., Stoddart, J.F.: Design and synthesis of glycodendrimers. Rev. Mol. Biotechnol. 90(3-4), 231-255 (2002)

17. Roy, R.: Glycodendrimers: a new class of biopolymers. Polym. News 21(7), 226-232 (1996)

18. Jayaraman, N., Nepogodiev, S.A., Stoddart, J.F.: Synthetic carbohydrate-containing dendrimers. Chem. Eur. J. 3(8), 1193 1199 (1997)

19. Branderhorst, H.M., Ruijtenbeek, R., Liskamp, R.M.J., Pieters, R.J.: Multivalent carbohydrate recognition on a glycodendrimerfunctionalized flow-through chip. Chembiochem 9(11), 18361844 (2008)

20. Parera Pera, N., Branderhorst, H.M., Kooij, R., Maierhofer, C., van der Kaaden, M., Liskamp, R.M.J., Wittmann, V., Ruijtenbeek, R., Pieters, R.J.: Rapid screening of lectins for multivalency effects with a glycodendrimer microarray. Chembiochem 11(13), 1896 $1904(2010)$

21. Zhou, X., Turchi, C., Wang, D.: Carbohydrate cluster microarrays fabricated on three-dimensional dendrimeric platforms for functional glycomics exploration. J. Proteome Res. 8(11), 5031-5040 (2009)

22. Fukuda, T., Onogi, S., Miura, Y.: Dendritic sugar-microarrays by click chemistry. Thin Solid Films 518(2), 880-888 (2009)

23. Spain, S.G., Gibson, M.I., Cameron, N.R.: Recent advances in the synthesis of well-defined glycopolymers. J Polym. Sci. A Polym. Chem. 45, 2059-2072 (2007)

24. Godula, K., Rabuka, D., Nam, K.T., Bertozzi, C.R.: Synthesis and microcontact printing of dual end-functionalized mucin-like glycopolymers for microarray applications. Angew. Chem. Int Ed. 48(27), 4973-4976 (2009)

25. Godula, K., Bertozzi, C.R.: Synthesis of glycopolymers for microarray applications via ligation of reducing sugars to a poly(acryloyl hydrazide) scaffold. J. Am. Chem. Soc. 132(29), $9963-9965$ (2010)

26. Lee, S.-G., Brown, J.M., Rogers, C.J., Matson, J.B., Krishnamurthy, C., Rawat, M., Hsieh-Wilson, L.C.: Endfunctionalized glycopolymers as mimetics of chondroitin sulfate proteoglycans. Chem. Sci. 1(3), 322-325 (2010)

27. Narla, S.N., Sun, X.-L.: Orientated glyco-macroligand formation based on site-specific immobilization of $\mathrm{O}$-cyanate chain-end functionalized glycopolymer. Org. Biomol. Chem. 9, 845-850 (2011)

28. Narla, S.N., Sun, X.-L.: Glyco-macroligand microarray with controlled orientation and glycan density. Lab Chip 12(9), 1656-1663 (2012)

29. Zhu, X.Y., Holtz, B., Wang, Y., Wang, L.-X., Omdorff, P.E., Guo, A.: Quantitative glycomics from fluidic glycan microarrays. J. Am. Chem. Soc. 131, 13646-13650 (2009)

30. Ma, Y., Zhang, H., Gruzdys, V., Sun, X.-L.: Azide-reactive liposome for chemoselective and biocompatible liposomal surface functionalization and glyco-liposomal microarray fabrication. Langmuir 27(21), 13097-13103 (2011)

31. Zhang, H., Ma, Y., Sun, X.-L.: Chemically selective liposome surface glyco-functionalization. Methods Mol. Biol. 751, 269-280 (2011)

32. Zhang, H., Ma, Y., Sun, X.-L.: Chemically-selective surface glycofunctionalization of liposomes through Staudinger ligation. Chem. Commun. 21, 3032-3034 (2009)

33. Zhang, Y., Campbell, C., Li, Q., Gildersleeve, J.C.: Multidimensional glycan arrays for enhanced antibody profiling. Mol. BioSyst. 6(9), 1583-1591 (2010)

34. Tong, Q., Wang, X., Wang, H., Kubo, T., Yan, M.: Fabrication of glyconanoparticle microarrays. Anal. Chem. 84(7), 3049-3052 (2012)

35. Matthews, O.A., Shipway, A.N., Stoddart, J.F.: Dendrimersbranching out from curiosities into new technologies. Prog. Polym. Sci. 23, 1-56 (1998) 
36. Yalong, Z., Gildersleeve, J.C.: General procedure for the synthesis of neoglycoproteins and immobilization on epoxide-modified glass slides. Methods Mol. Biol. 808, 155-165 (2012)

37. Kerékgyártó, M., Fekete, A., Szurmai, Z., Kerẻkgyártó, J., Takảcs, L., Kurucz, I., Guttman, A.: Neoglycoproteins as carbohydrate antigens: synthesis, analysis, and polyclonal antibody response. Electrophorosis 34, 2379-2386 (2013)

38. Narla, S.N., Nie, H., Li, Y., Sun, X.-L.: Recent advances in the synthesis and biomedical applications of chain-end functionalized glycopolymers. J. Carbohyr. Chem. 31(2), 67-92 (2012)

39. Godula, K., Bertozzi, C.R.: Density variant glycan microarray for evaluating cross-linking of mucin-like glycoconjugates by lectins. J. Am. Chem. Soc. 134, 15732-15742 (2012)

40. Bian, S., Zieba, S.B., Morris, W., Han, X., Richter, D.C., Brown, K.A., Mirkin, C.A., Braunschweig, A.B...: Beam pen lithography as a new tool for spatially controlled photochemistry, and its utilization in thesynthesis of multivalent glycan arrays. Chem. Sci. 5, 2023-2030 (2014)

41. Chan, Y.-H.M., Boxer, S.G.: Model membrane systems and their applications. Curr. Opin. Chem. Biol. 11(6), 581-587 (2007)

42. Denning, E.J., Beckstein, O.: Influence of lipids on proteinmediated transmembrane transport. Chem. Phys. Lipids 169, 5771 (2013)

43. Chrai, S., Murari, R., Imran, A.: Liposomes: a review. BioPharm 14(11), 10-14 (2001)

44. Akbarzadeh, A., Rezaei-Sadabady, R., Davaran, S., Joo, S.W., Zarghami, N., Hanifehpour, Y., Samiei, M., Kouhi, M., NejatiKoshki, K.: Liposome: classification, preparation, and applications. Nanoscale Res. Lett. 8, 102-110 (2013)

45. Patil, Y.P., Jadhav, S.: Novel methods for liposome preparation. Chem. Phys. Lipids 177, 8-18 (2014)

46. Tamm, L.K., McConnell, H.M.: Supported phospholipid bilayers. Biophys. J. 47(1), 105-113 (1985)

47. Granéli, A., Rydström, J., Kasemo, B., Höök, F.: Formation of supported lipid bilayer membranes on $\mathrm{SiO}_{2}$ from proteoliposomes containing transmembrane proteins. Langmuir 19(3), 842-850 (2003)

48. Cremer, P.S., Boxer, S.G.: Formation and spreading of lipid bilayers on planar glass supports. J. Phys. Chem. B 103(13), 2554-2559 (1999)

49. Richter, R.P., Brisson, A.R.: Following the formation of supported lipid bilayers on mica: a study vombining AFM, QCM-D, and ellipsometry. Biophys. J. 88(5), 3422-3433 (2005)
50. Richter, R.P., Bérat, R., Brisson, A.R.: Formation of solidsupported lipid bilayers: an integrated view. Langmuir 22(8), 3497-3505 (2006)

51. Tabaei, S.R., Jönsson, P., Brändẻn, M., Hook, F.: Self-assembly formation of multiple DNA-tethered lipid bilayers. J. Struct. Biol. 168(1), 200-206 (2009)

52. Silin, V.I., Wieder, H., Woodward, J.T., Valincius, G., Offenhausser, A., Plant, A.L.: The role of surface free energy on the formation of hybrid bilayer membranes. J. Am. Chem. Soc. 124, 14676-14683 (2002)

53. Terrettaz, S., Mayer, M., Vogel, H.: Highly electrically insulating tethered lipid bilayers for probing the function of ion channel proteins. Langmuir 19, 5567-5569 (2003)

54. Munro, J.C., Frank, C.W.: In situ formation and characterization of poly(ethylene glycol)-supported lipid bilayers on gold surfaces. Langmuir 20(24), 10567-10575 (2004)

55. Wagner, M.L., Tamm, L.K.: Reconstituted syntaxin1A/SNAP25 interacts with negatively charged lipids as measured by lateral diffusion in planar supported bilayers. Biophys. J. 81(1), 266-275 (2001)

56. Goennenwein, S., Tanaka, M., Hu, B., Moroder, L., Sackmann, E.: Functional incorporation of integrins into solid supported membranes on ultrathin films of cellulose: impact on adhesion. Biophys. J. 85, 646-655 (2003)

57. Yoshina-Ishii, C., Boxer, S.G.: Arrays of mobile tethered vesicles on supported lipid bilayers. J. Am. Chem. Soc. 125(13), 3696-3697 (2003)

58. Svedhem, S., Pfeiffer, I., Larsson, C., Wingren, C., Borrebaeck, C., Höök, F.: Patterns of DNA-labeled and scFv-antibody-carrying lipid vesicles directed by material-specific immobilization of DNA and supported lipid bilayer formation on an $\mathrm{Au} / \mathrm{SiO}_{2}$ template. Chembiochem 4(4), 339-343 (2003)

59. Ma, Y., Sobkiv, I., Gruzdys, V., Zhang, H., Sun, X.-L.: Liposomal glyco-microarray for studying glycolipid-protein interactions Anal. Bioanal. Chem. 404, 51-58 (2012)

60. Murthy, S.K.: Nanoparticles in modern medicine: state of the art and future challenges. Int. J. Nanomed. 2(2), 129-141 (2007)

61. Marradi, M., Chiodo, F., García, I., Penadès, S.: Glyconanoparticles as multifunctional and multimodal carbohydrate systems. Chem. Soc. Rev. 42(11), 4728-4745 (2013) 